\title{
Channel modeling and power consumption analysis for galvanic coupling intra-body communication
}

\author{
Yue Ming Gao ${ }^{1,2^{*}}$, Yan Ting Ye ${ }^{1,2}$, Mang I Vai ${ }^{2,3,4}$, Min Du ${ }^{1,2}$ and Sio Hang Pun ${ }^{3,2}$
}

\begin{abstract}
Intra-body communication (IBC), using the human body as the channel to transmit data, has lower power consumption, less radiation, and easier linking than common wireless communication technologies such as Bluetooth, ZigBee, and $\mathrm{ANT}^{+}$. As a result, IBC is greatly suitable for body area network (BAN) applications, such as the medical and health care field. Furthermore, IBC can be implemented in wearable devices, including smart watches, sports bracelets, somatic game devices, and multimedia devices. However, due to the limited battery capacity of sensor nodes in a BAN, especially implanted sensor nodes, it is not convenient to charge or change the batteries. Thus, the energy effectiveness of the media access control (MAC) layer strongly affects the life span of the nodes and of the entire system. Certainly, analyzing MAC layer performance in a galvanic coupling IBC is of great importance for the overall system. To obtain the attenuation properties of IBC, in vivo experiments with seven volunteers were performed. Meanwhile, an equalizer was used to compensate the frequency distortion in consideration of frequency-selective fading characteristics of intra-body channels. In addition, a comparison of the bit error rates (BER) of different modulation methods was carried out to obtain the best modulation method. Then, the attenuation characteristics of intra-body channels were applied in a multi-node physiological signal monitor and transmission system. Finally, TDMA and CSMA/CA protocols were introduced to calculate the bit energy consumption of IBC in the practical scenario. With stable characteristics of the intra-body channels, QPSK with an equalizer had a better performance than the tests without an equalizer. As a result, the modulation method of FSK could achieve a lower BER in lower signal-to-noise ratio situations and an FSK method with TDMA for the IBC had the lowest energy consumption under different practical scenarios.
\end{abstract}

Keywords: FSK, IBC, Human channel mode, Modulation methods, Equalizer, TDMA, CSMA/CA

\section{Introduction}

Nowadays, with the development of economy and technology, there is a boom in wearable devices, such as smart watches, smart wristbands, sports bracelets, somatic game devices, and multimedia devices. Generally, these wearable devices were connected through Bluetooth or ZigBee. To increase the integration and communication security, body area network (BAN) was proposed as a new generation of wireless sensor networks which works in the vivo and vitro of the human

\footnotetext{
*Correspondence: yueminggaomtap@163.com

'College of Physics and Information Engineering, Fuzhou University, Fuzhou, China

${ }^{2}$ Key Lab of Medical Instrumentation \& Pharmaceutical Technology of Fujian Province, Fuzhou, China

Full list of author information is available at the end of the article
}

body. It could be applied in many domains, including health care, medical, military, and consumer electronics [1]. Honeine, P. et al. [2] indicated using BAN in health care and medical domains to monitor the vital physiological signals. They analyzed the long-term information of physiological sensors which were worn or implanted in the body to provide a health care for some common diseases, such as diabetes, hypertension, and heart attack. In a health care BAN, transmission method should be low power consumption, low radiation, and high transmission efficiency and security. However, with high power consumption and network insecurity, traditional communication technologies (Bluetooth or ZigBee) were not suitable for the BAN. In this regard, intra-body communication (IBC) was identified by IEEE Standards 
Organization as a physical layer standard for BAN [3]. In intra-body communication (IBC), human body was selected as transmission medium for electrical signal propagation [4]. There are two signal coupling types in IBC, which are capacitive coupling and galvanic coupling, respectively [4-8]. In capacitive coupling IBC, the signal channel is mainly composed of four parts-human body, signal electrode, ground electrode, and the external ground. The ground electrode couples the signal towards the external ground via the electrostatic field. Meanwhile, the surrounding environment has a great influence on the quality of communication, whereas galvanic coupling injects the electric current signal into the body through a pair of electrodes attached to the skin, and another pair of electrodes is used to differentially detect the coupling potential on the skin. The transmission path of galvanic coupling IBC is completely dependent on the human body. Therefore, it does not interact with the external environment which contributes to higher security.

In galvanic coupling IBC, the human body is utilized as the data channel to carry information [4]. The signal transmission is implemented by coupling current signals into the body and picking up the surface potential differentially by two pairs of transceiver electrodes which are attached to the human body. Certainly, human body channel characteristics play a significant role in the data transmission of IBC. The signal attenuations and BER under different carrier frequencies and modulation methods are obtained. Obviously, there are frequency distortions and inter-symbol interference (ISI) existed in human body channels. Thus, the channel model, modulation method, and equalizer should be investigated to reduce the $B E R$ and increase the band efficiency of an IBC system.

\section{Related works}

Recently, several papers have reported on IBC investigation. The concept of IBC was initially proposed by Zimmerman in 1995, following the first circuit model for the communication channel of the body [4]. M. Amparo Callejon et al. [9] proposed a simple model based on a distributed parameter structure which flexibly adapts to both galvanic and capacitive coupling. Furthermore, they verified the effectiveness of the model through an experiment with two methods. Then, the human body channel model was equivalent to a fourterminal circuit to be designed and analyzed by in vivo experiment [10]. M. S. Wegmueller et al. [11] compared the different electrodes and designed a testing system with up to $1 \mathrm{~mA}$ contact current modulated in the frequency range of $10 \mathrm{kHz}$ to $1 \mathrm{MHz}$. In [11], they proved that galvanic coupling of IBC was lower power consumption than other wireless technologies. Željka Lucev et al. [12] analyzed the capacitive IBC channel transmission characteristics in the frequency range from $100 \mathrm{kHz}$ to $100 \mathrm{MHz}$. In [12], they used different electrode arrangements, test persons, environments, and body positions and movements. They also used a network analyzer and a pair of baluns to obtain the reliability characteristics of a realistic, capacitive IBC channel. N. Haga et al. [13] proposed a theory of the equivalent circuit for lossy conductors and addressed the physical mechanism of the communication channels as well. In China, Ruoyu Xu et al. [14] established an electric-field IBC model based on a finite element method (FEM) model, and studied environmental effects on the electric-field intra-body communication channel. Wang Hao et al. [15] designed a high-speed IBC receiver, which concentrated on high data speed, 2.5 and $5 \mathrm{Mbit} / \mathrm{s}$, extremely long transmission distances, $170 \mathrm{~cm}$, and applied on an FPGA-based audio player. In [15], by a simple touch on the transmitter electrode, the data would be sent through one hand to the other hand attached by a receiver electrode. Changjiang Dua, Zedong Nie et al. invented a voice communication system based on human communication $[16,17]$. It included an audio transmission device and an audio reception device. The human body was employed as an audio signal transmission medium, where the audio signal transmission was near the ear, and an audio reception device in the ear received and played the audio signal from the human body. This audio communication system used IBC technology and had the advantages of low power consumption, high confidentiality, and less body damage. Xi Mei Chen et al. [18] studied the IBC channel characteristics through a comparison between theoretical calculations via transfer functions and experimental measurements in both the frequency domain and the time domain. In [18], Lysis versus different transmission distances. Harmonic distortions were analyzed in both base-band and pass-band transmissions for square input waves. They also explored the BER performance of several common modulation schemes in an IBC system with a carrier frequency of $500 \mathrm{kHz}$.

The aforementioned researches of IBC channel characteristics were mainly focused on the design of channel circuit models, the experimental design, data analysis, and the electromagnetic characteristics of human tissues. However, they didn't consider the pros and cons of various modulation methods and the ISI of IBC channel. The research of energy efficiency and low power consumption for a wireless body area network (WBAN) started at the end of the 20th century. In 2008, Omeni et al. [19] firstly studied the problem of energy efficiency of WBAN from the viewpoint of protocol, and they proposed a new MAC protocol of energy efficiency. In 2012, Prabh [20] achieved time synchronization and combination with the existing TDMA protocol by its 
own electrocardiogram (ECG) signal, and proposed a BAN MAC, which was another breakthrough of MAC design for WBAN. In 2013, S. Hayat [21] proposed an energy-efficient MAC protocol for WBAN. At the same year, Ramona Rosini [22] researched channel measurement and MAC performance evaluation on the surface of the human body in a WBAN. The majority of MAC protocols were based on TDMA or CSMA/CA according to previous researches on MAC protocols. A multi-node physiological signal monitor and transmission system using the channel characteristics of IBC were assumed as the application scenario, and two common protocols were chosen to calculate and compare the bit energy consumption of IBC at the same application situation.

In this view, this paper focused on the study of the channel model of IBC systems. The rest of this paper is organized as follows. With the signal analyzer in a constant voltage circuit and a differential probe, we did in-vivo experiments with seven volunteers to obtain the amplitudefrequency characteristics of human body channel. Then, a band-pass filter was designed based on the experiment data to serve as the model of a human body channel. Based on the channel characteristics in Section 3, an equalizer was designed to compensate for the frequency distortion of the human body channel. Following this, BER of different kinds of modulation methods in an IBC base band modulation simulation model were evaluated and compared. QPSK constellation diagrams and the performance of the equalizer were investigated in Section 4 as well. An intra-body communication transceiver based on an FSK method is proposed in Section 5. The transmitter consists of a micro-controller and a modulation circuit, while the receiver contains an AFE (analog front end) which involves a conditioning circuit (amplifier, filter, and comparator), demodulation circuit, and display section. The bit energy consumption of two MAC protocols-TDMA and CSMA/CA, were calculated and compared in an IBC application scenario in Section 6. Finally, the conclusions were drawn in Section 7.

\section{Channel modeling of the human body}

In order to obtain the attenuation properties of the human body channel, in-vivo experiments employing the forearms of 7 volunteers were carried out. As shown in Fig. 1, the experiment system was mainly composed of three parts-a spectrum analyzer, a constant voltage circuit, and differential probe. The transmission characteristics of the IBC system were measured in a frequency between $10 \mathrm{kHz}$ and $500 \mathrm{kHz}$. To stabilize voltage amplitude of the output scanning signal from the spectrum analyzer in a certain range, a constant voltage circuit was designed. Then the signal was injected into the human body by transmitter electrodes. Through an Agilent 1141 differential probe, the receiver received the signal and send it to the spectrum analyzer. Battery supply was applied to the signal follower and differential probe to suppress $50 \mathrm{~Hz}$ power line interference and remove the effect of the internal ground of the transceiver. During the measurement, physiotherapy electrodes $(4 \mathrm{~cm}$ by $4 \mathrm{~cm}$ in area) were used for coupling the signal into the human body. At the beginning, this paper used the forearms of 7 volunteers as experiment subjects and within the scope of the forearm length, human body characteristic curves were basically the same. In this regard, the

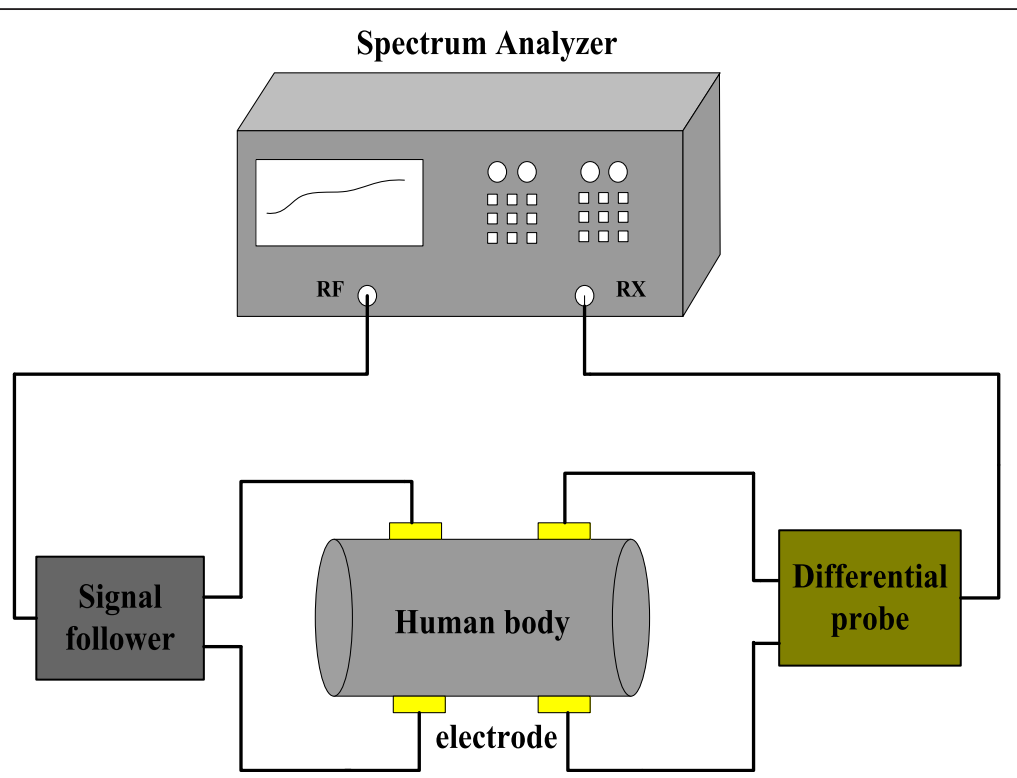

Fig. 1 Measurement system of intra-body communication, composed of spectrum analyzer, signal follower (transmitter constant voltage circuit) and differential probe (receiver) 
distance between transceivers was set to $10 \mathrm{~cm}$ in this experiment.

As shown in Fig. 2, the measurement results show that different volunteers had the same attenuation characteristics in the frequency range of $10-500 \mathrm{kHz}$. Besides, the thicker forearms had lower attenuation, which indicating that the main path of the IBC signals was muscle [23]. Additionally, the suitable frequency band of transmission signals for the human body was relatively concentrated between $20-50 \mathrm{kHz}$ which could infer that human body channel characteristics approximated those of a band-pass filter in the range of $20-50 \mathrm{kHz}$.

The mean value of the in-vivo experimental data and the approximate computation in the simulation tool of MATLAB/SIMULINK [24-26], which was based on several key points of in-vivo experimental data, were utilized to design a band-pass filter. Amplitude-frequency characteristics of the filter and experimental data were shown in Fig. 3. The correlation coefficient of experimental data and the fitting curve approached 0.9989, which indicating that the human body channel model was well matched with the in-vivo experiment.

As shown in Fig. 3, there is lower attenuation of amplitude in the frequency band from $10 \mathrm{kHz}$ to $100 \mathrm{kHz}$ which can be assumed as a pass band that is suitable for data transmission. In order to estimate the capacity of galvanic coupling body channels, the signal-noise ratios of different frequency signals in the $10 \mathrm{~cm}$ body channel was measured by a bi-polar sine wave signal whose transmitting power was $6.2 \mathrm{dBm}$, results as shown in Table 1.

Every sub-band that corresponds with its frequency point is assumed to be a flat fading band, which means that every sub-channel gain does not change by time. The expression of Shannon channel capacity based on water-filling theory is shown as follows:

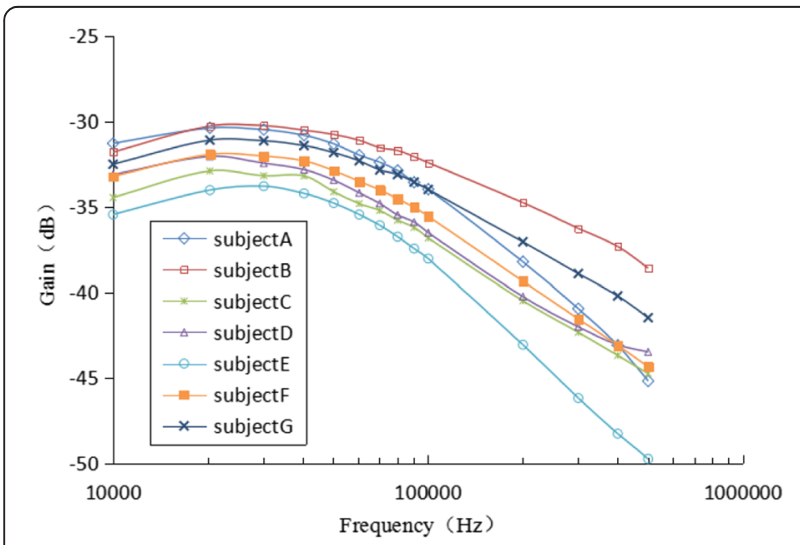

Fig. 2 Attenuation characteristics of the forearm of seven volunteers

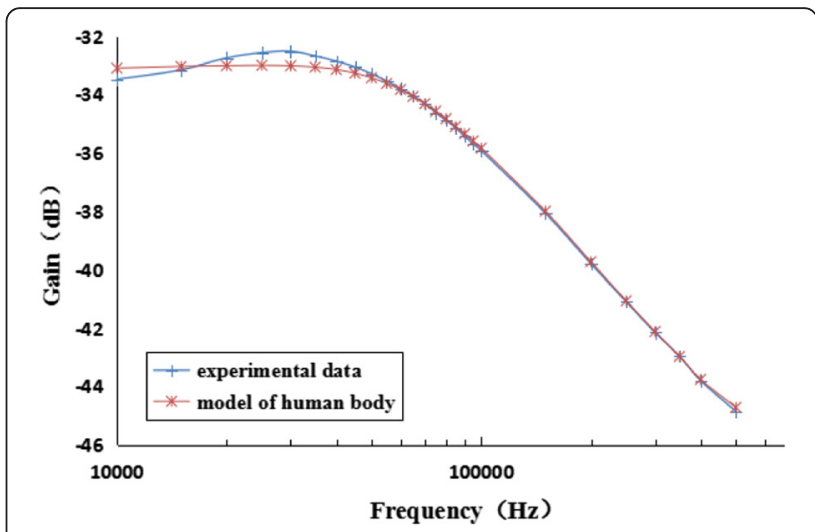

Fig. 3 Comparison of amplitude-frequency characteristic of the model and in vivo experiment

$$
\begin{aligned}
& C_{i}=B_{i} \log _{2}\left(1+S N R_{i}\right) \\
& C=\sum_{10}^{i=1} C_{i}
\end{aligned}
$$

According to (1) and (2), the estimated channel capacity of galvanic coupling intra-body communication is $412 \mathrm{Kbit} / \mathrm{s}$.

\section{IBC base band modulation simulation model}

BER and band efficiency are the main factors employed to measure the system performance. The application of efficient digital modulation can improve the performance of IBC systems. In this section, we report how we evaluated and compared different kinds of modulation-demodulation methods in a base band modulation simulation model of IBC that is based on the proposed human body channel model in Section 3 and use of a MATLAB/SIMULINK tool. Proakis et al. [27] states that in the frequency range up to $1 \mathrm{kHz}$, biological signal interference is very weak and can be ignored in the human body. Thus, an IBC channel is equivalent to an AWGN channel. As shown in Fig. 4, a base band modulation simulation model was composed of the signal generator, modulator-demodulator, and human body channel with AWGN noise and an equalizer module.

According to the frequency spectrum characteristics of the IBC channel in Fig. 3, there is certainly frequency distortion and ISI existed in the human body channel. Therefore, in order to reduce BER of an IBC system, an equalizer was designed to compensate for the frequency distortion. Based on the no ISI conditions of the Nyquist first principles, if the sum of channel amplitude-frequency characteristic $H(f)$ and equalizer amplitude-frequency characteristic $E(f)$ was a horizontal line, distortion and BER would be reduced [28].

$$
H(f) * E(f)=1
$$

So, the amplitude-frequency characteristic of equalizer $E(f)$ was as follows: 
Table 1 Signal-noise ratio of different frequency signal

\begin{tabular}{lllllllllll}
\hline$f(\mathrm{kHz})$ & 10 & 20 & 30 & 40 & 50 & 60 & 70 & 80 & 90 & 100 \\
SNR (dB) & 9.2 & 22.4 & 22.4 & 14.8 & 20.9 & 14.3 & 18.6 & 18.5 & 13.7 & 14.5 \\
Sub-band $(\mathrm{kHz})$ & $5-14$ & $15-24$ & $25-34$ & $35-44$ & $45-54$ & $55-64$ & $65-74$ & $75-84$ & $85-94$ & $95-104$ \\
\hline
\end{tabular}

$$
E(f)=\frac{1}{H(f)}
$$

According to (4), the mean value of the experimental data was used to obtain the characteristic parameters of the equalizer. Figure 5 shows the design of the filter $E(f)$. The equalizer acted as a signal pre-processing circuit including amplifier, filter, and reshaper, in the implementation of the IBC system.

Random Integer Generator mode of MATLAB/ SIMULINK tool was used to generate binary signal, in which data rate was $10 \mathrm{kbps}$, and the binary signal through the IBC base-band modulation simulation system and then demodulation output. Considering the complexity of IBC systems, modulations of FSK, BPSK, QPSK, and 8PSK were selected. Error Rate Calculation mode of MATLAB/SIMULINK tool was used to analyze the BER of four kinds of modulation method under different SNR. BER versus SNR in four kinds of modulation methods with equalizer (a) and without equalizer (b) with the range in SNR from 2 to 18 is shown in Fig. 6.

Figure $6 a$ indicated that the BER and band efficiency was considered at the same time. FSK could attain the lowest BER for the IBC system, whereas the band efficiency of FSK was lower than PSK. However, when the SNR was higher than $8 \mathrm{~dB}$, the BER of 8PSK and QPSK were similar. In terms of band efficiency, QPSK is twice as high as of BPSK's. The 8PSK had the highest BER of these modulation methods as well as higher band efficiency at the same time. Thus, when it comes to requiring higher band utilization, FSK had a lower BER and could better conform to the requirements of an IBC system and FSK was not affected by a change in channel parameters.

As shown in Fig. 6a, b, the equalizer significantly reduced the BER of the human body channel. Furthermore, the constellation diagrams were greatly improved, as shown in Fig. 7, which means that the equalizer could effectively reduce the ISI induced by the frequency distortion of the human body channel.

\section{Implementation of an intra-body communication system}

According to Fig. 6, we can conclude that the FSK method has a lower BER when compared to other modulationdemodulation methods in intra-body communication, and therefore, it is much more suitable to be applied in data transmission by intra-body communication system. Then use of an intra-body communication transceiver based on a FSK modulation-demodulation method is proposed in this section. The transmitter of the transceiver consists of a micro-controller and modulation circuit, while the receiver contains an AFE (Analog Front End) which involves a conditioning circuit (amplifier, filter, and comparator) a demodulation circuit, and a display section, as shown in Fig. 8.

In addition, the implementation of the intra-body communication system is shown in Fig. 9, and parameters of the system are shown in Table 2.

As shown in the Fig. 9, (1) represents the receiver signal, and (2) represents the transmission signal. As a result of the existence of transmission distance and the low data rate, the transmitted signal and the receiver signal exhibit a phase delay. The experimental results show that the IBC system can realize transmission signal.

In this section, an intra-body communication system of point-to-point was implemented. But in the application scenario of medical BAN, multi-node application scenario and the energy effectiveness of system are necessary to be analyzed. Due to the limited battery capacity of sensor nodes in a BAN, especially for implanted sensor nodes, it is not convenient to charge or change the batteries. Therefore, the energy effectiveness of the media access control (MAC) layer strongly affects the life span of the nodes and of the entire system. Certainly, analyzing MAC layer performance in a galvanic coupling IBC is of great importance for the overall system.

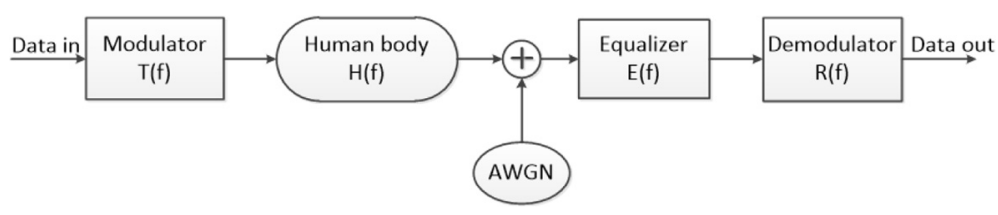

Fig. 4 IBC base band modulation simulation model 


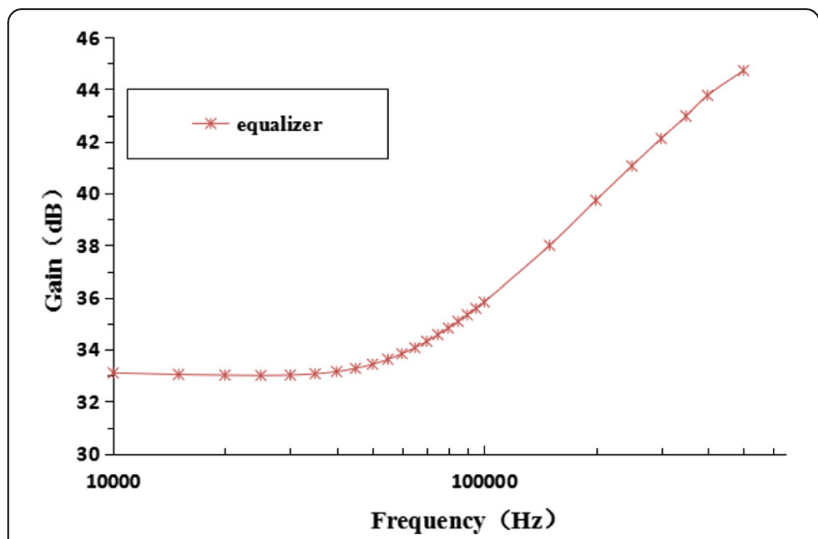

Fig. 5 Amplitude-frequency characteristic of equalizer $E(f)$

\section{The energy consumption of the MAC layer}

The last section implemented the point-to-point intrabody communication system, and this section focuses on the choice of two common protocols in a multi-node galvanic coupling IBC system.

To investigate the energy consumption of MAC layer based on TDMA and CSMA/CA, using the proposed band-pass filter characteristics of the human body in section 3, designed a multi-node physiological signal monitor and transmission system using a star topology at the fist. The modulation method took advantage of the lower BER of FSK. Each node acquired the signal by STM32 with 12-bit ADC in this system and transmitted to the master node via the galvanic coupling IBC. In order to complete the protocol system function for the medical BAN application, TDMA and CSMA/CA protocols were introduced to calculate the bit energy consumption in the practical scenario.

\subsection{TDMA}

TDMA protocol divides the timeline into different time slots [29], so that multiple users in different time slots to use the same frequency. The mechanism is very suitable for the limited channel bandwidth of galvanic coupling
IBC. A multi-node transmission system of star topology structure using IBC and TDMA is assumed as follows: the master node sends the control frame within the special ID to all sub-nodes in the form of polling. One subnode with the same ID will reply to the master. Since the electromagnetic wave transmission time is far less than the time the node data requires in the human body, the signal transmission time in the human body can be ignored. The system transmission bit number is assumed as the constant $c$; then the packet number $m$ can be represented as follows:

$$
m=c /\left(8 \times S_{d s}\right)
$$

here $S_{d s}$ denotes the data frame length.

In this multi-node IBC system, $S_{t m}$ denotes the polling data frame length of the master node, and $r$ is the data throughput of IBC process. The time when the master node sends the control frame is $T_{t m}$ :

$$
T_{t m}=S_{t m} / r
$$

When the sub-node receives the request frame, it responds to the master node. Let $S_{c s}$ and $S_{d s}$ be the control segment and the data segment of the response frame, and the time when the sub-node sends a response frame to the master node is $T_{t s}[30,31]$.

$$
T_{t s}=\left(S_{d s}+S_{c s}\right) / r
$$

Let us suppose that the average bit error rate of the IBC system is BER. In case of packet loss, the master node would re-transmit the packet. So the success probability of a data packet can be calculated as follows:

$$
p=(1-B E R)^{S_{p} \times 8}
$$

where $S_{p}$ is the data packet in bytes of transmission. Assume that the node enters into standby mode when not required to send and receive data, then when combined with equations (5), (6), (7), and (8), the energy consumption of sub-nodes in successful transmission of one data packet:
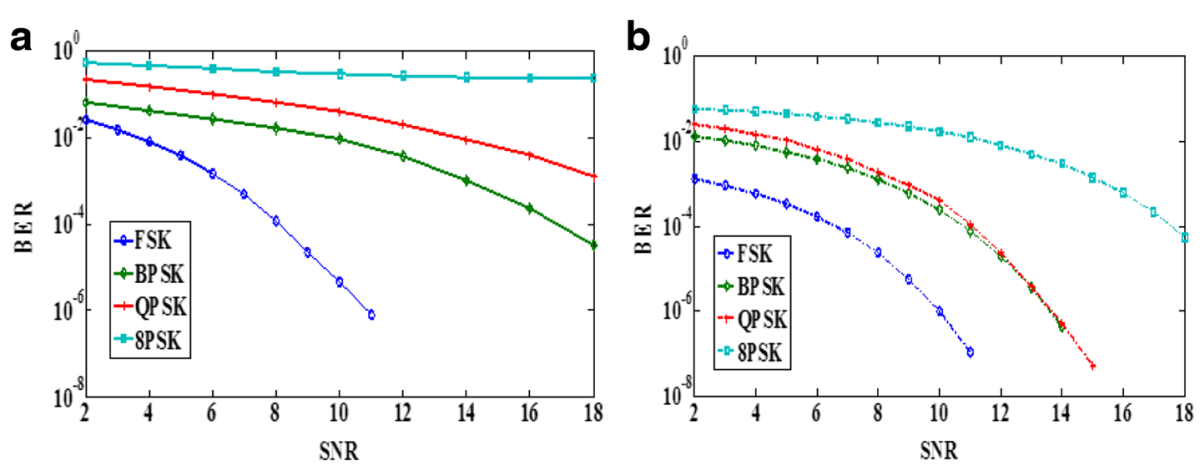

Fig. 6 BER versus SNR of four kinds of modulation method with equalizer (a) and without equalizer (b) 

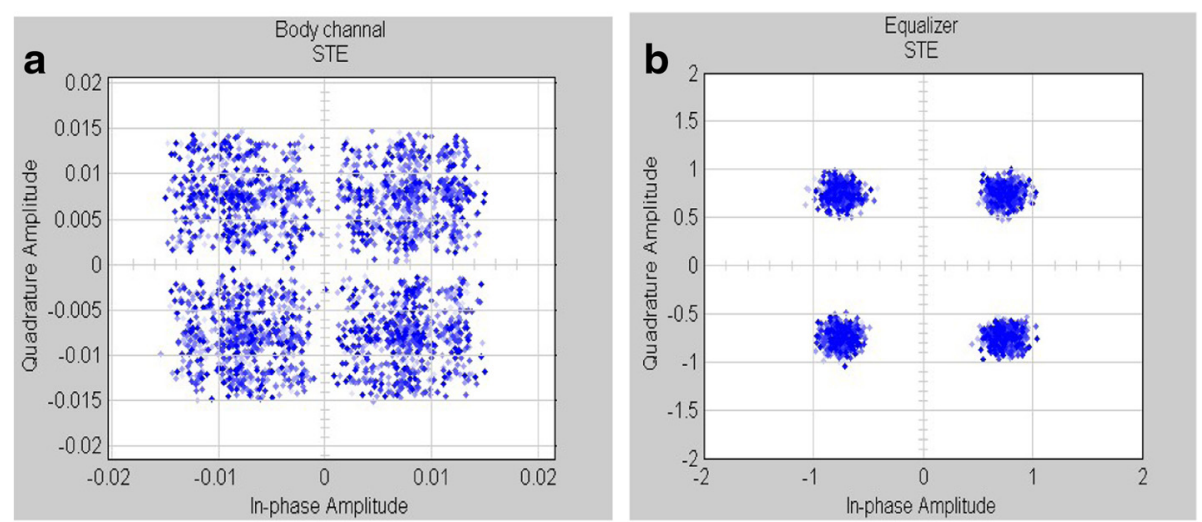

Fig. 7 Constellation diagrams of QPSK without the equalizer (a) and with the equalizer (b)

$$
E_{t 1}=\left[N \times P_{r} \times T_{t m} \times \frac{1}{p^{2}}+P_{t} \times T_{t s} \times \frac{1}{p}+P_{i d} \times T_{t s} \times(N-1)\right] \quad E_{t c}=E_{t} / c
$$

Among these terms, $P_{n}, P_{t}$, and $P_{i d}$ are the power drawn by the receiver, transmitter, and in the idle state, respectively. The first item of Eq. (9) represents the energy consumption for reception of the polling frame of $\mathrm{N}$ sub-nodes. The second one is the consumption of transmitting data of the specified node. The last item is the idle state energy consumption for the remaining $(\mathrm{N}-1)$ nodes.

For the master node, the energy consumption for network transmission of a packet is as follows:

$$
E_{t}=\left(E_{t 1}+E_{t 2}\right) \times m
$$

Therefore, when all the data is sent successfully, the total energy consumption is calculated as follows:

$$
E_{t}=\left(E_{t 1}+E_{t 2}\right) \times m
$$

Applying formulas (5), (6), (7), (8), (9), (10), and (11), the bit energy consumption $E_{t c}$ of the TDMA system is available as follows:

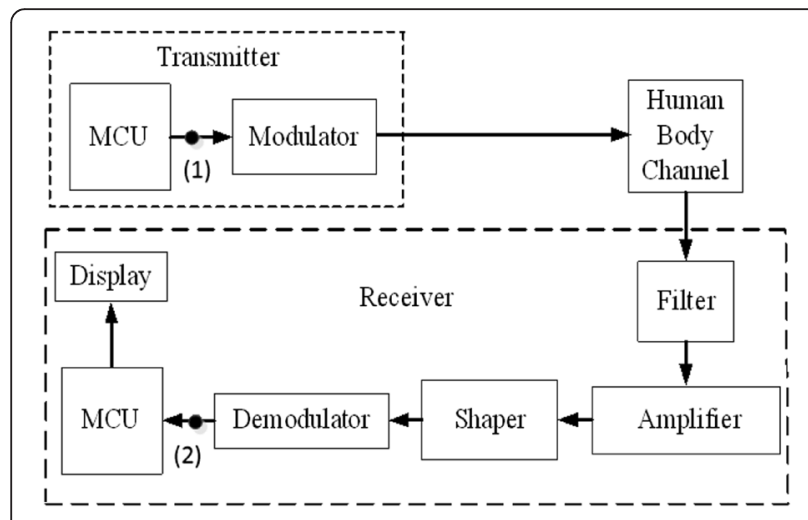

Fig. 8 Diagram of intra-body communication

\subsection{CSMA/CA}

CSMA/CA is a network multiple access method in which carrier sensing is used [29]. The network nodes attempt to avoid collisions by transmitting their packet data in entirety only when the channel is sensed to be "idle." This collision avoidance mechanism is beneficial to avoid the data loss of certain vital physiological signals in a medical BAN when, for instance, an emergency has occurred. The master node broadcasts a control segment with the packet size information. Each sub-node receives the request and sends a corresponding size data packet to the master through the use of CSMA/CA.

The symbols $S_{d s}, S_{c s}, r, c$, and $p$ represent the same parameters as in the case of TDMA. The polling request frame length of the master node is denoted by $S_{c m}$. So the time used to send the polling frame $T_{c m}$ is defined as follows:

$$
T_{c m}=S_{c m} / r
$$

The back-off window size CW1 is defined for the subnode with the unit ms. The maximum re-transmission number is $R$. Then the average back-off time for listening one packet is shown as follows:

$$
t_{c} \frac{C W_{1}}{2} \times \min \left\{\frac{N-1}{2}, R\right\}
$$

In most of the existing CSMA/CA applications [31], $C W_{1}$ is equal to $2.5 \mathrm{~ms}$. The energy consumption of $\mathrm{N}$ sub-nodes in succeed transmit one data packet is as follows: 


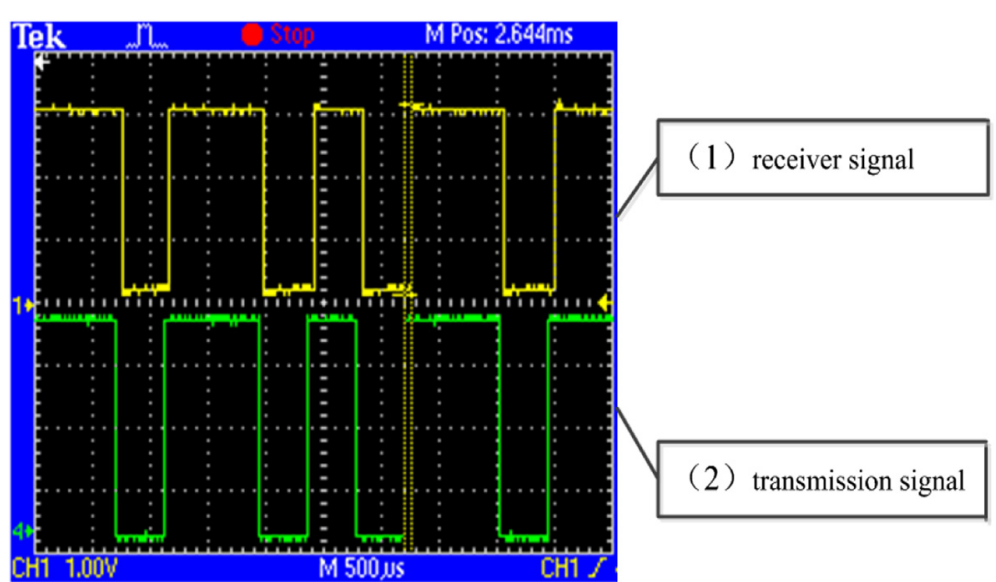

Fig. 9 Implementation of intra-body communication: (1) represents the receiver signal, and (2) represents the transmission signal

$$
\begin{aligned}
E_{c 1}= & {\left[N \times P_{r} \times T_{c m} \times \frac{1}{p^{2}}+\left(P_{t} \times T_{c s}+N \times P_{c} \times t_{c}\right)\right.} \\
& \left.\times \frac{1}{p}+P_{i d} \times t_{d} \times \frac{1}{p} \times(N-1)\right]
\end{aligned}
$$

Energy consumption of master nodes successfully transmitting one data packet is as follows:

$$
E_{c 2}=\left[P_{t} \times T_{c m} \times \frac{1}{p^{2}}+P_{r} \times T_{c s} \times \frac{1}{p}+P_{i d} \times t_{c} \times \frac{1}{p}\right]
$$

The whole network energy consumption of one cycle is as follows:

$$
E_{c}=\left(E_{c 1}+E_{c 2}\right) \times m
$$

From (13), (14), (15), (16), and (17), the bit energy consumption of CSMA/CA protocol is available:

$$
E_{c c}=E_{c} / c
$$

\subsection{Results}

Based on the description of the application scenarios and the equations deducing the bit energy consumption, the parameters of TDMA and CSMA/CA were set as shown in Table 3. The transmission power $P_{t}$, received

Table 2 Parameters of Transceiver

\begin{tabular}{ll}
\hline & Parameter \\
\hline Method & FSK \\
Bit rate & $2.4 \mathrm{Kbit} / \mathrm{s}$ \\
Carrier frequency & $20 \mathrm{kHz}, 40 \mathrm{kHz}$ \\
amplitude & $3.3 \mathrm{~V}$ \\
polarity & Uni-polarity \\
Channel length & $10 \mathrm{~cm}$ \\
\hline
\end{tabular}

power $P_{n}$ and listener power $P_{l}$ of the multi-node system were measured by the power analyzer and signal analyzer (Agilent N6705B and CXA N9000A). Each of them was assigned a maximum power value. $P_{i d}$ and $P_{s l}$ were measured in the different low power modes of STM32 [31]. Using the Fig. 6a simulation results, the BER of FSK was $10^{-6}$ when the SNR was $10 \mathrm{~dB}$. The response frame of the control segment $S_{c s}$ was 7 bytes, and the length of the packet $S_{d s}$ was 40 bytes. Due to 12-bit ADC of the detection, there were 2byte data for each sample. The sampling rate was set as $500 \mathrm{~Hz}$. Each node in the system transmitted 60-s sample data in total at one time through the galvanic IBC. Thus, the bit number $c$ in Eq. (5) to be transmitted from the sub-node to the master node was equal to $500 \times$ $16 \times 60$. The maximum data rate $r$ under the FSK modulation method for galvanic coupling IBC was, therefore, $50 \mathrm{kbit} / \mathrm{s}$.

Table 3 The parameters of IBC based on TDMA and CSMA/CA

\begin{tabular}{ll}
\hline Parameters & Value \\
\hline$P t(m W)$ & 10 \\
$P r(m W)$ & 10 \\
$P c(m W)$ & 10 \\
$P i d(m W)$ & 2 \\
$P S I(m W)$ & 1 \\
$S_{t m}($ byte $)$ & 15 \\
$S_{c s}($ byte $)$ & 7 \\
$S_{d s}($ byte $)$ & 40 \\
$S_{c m}($ byte $)$ & 15 \\
$R$ (kbps) & 50 \\
$C W_{l}(m s)$ & 2.5 \\
$R$ & 5 \\
$T(s)$ & 60 \\
\hline
\end{tabular}


The calculation results of 1-bit energy consumption of the IBC multi-node transmission system with numbers of sub-nodes from 2 to 15 based on TDMA and CSMA/CA are shown in Fig. 10. Firstly, the bit energy consumption of the CSMA/CA and TDMA became larger with the sub-node numbers increasing. When the node numbers were low, especially smaller than 4 , the energy consumption of the two protocols was smaller than $1.3 \mu \mathrm{J}$. When the sub-node numbers became larger, the power consumption of CSMA/CA increased rapidly. Yet the energy consumption of TDMA changed only a little along with the increasing of the sub-node numbers. For example, the CSMA/CA bit energy consumption was $1.3 \mu \mathrm{J}$ at 4 sub-nodes. However, its bit energy consumption was $3.1 \mu \mathrm{J}$ when the number of sub-nodes was 10 . The bit energy consumption of TDMA has the same general trend but with smaller increases. The TDMA bit energy consumption was $1.0 \mu \mathrm{J}$ at 4 sub-nodes. The bit energy consumption increased only to $1.5 \mu \mathrm{J}$ when the number of sub-node was 10 . Secondly, in a multinode transmission system, the bit energy consumption of TDMA was obviously lower than that of CSMA/CA. Further, we found that the gap of bit energy consumption for the two protocols increases gradually along with the increases in the sub-node numbers. For example, the CSMA/CA bit energy consumption was $1.85 \mu \mathrm{J}$ and the TDMA bit energy consumption was $1.2 \mu \mathrm{J}$, both at 6 sub-nodes-a difference of $0.65 \mu \mathrm{J}$. But when the number of sub-nodes was 14, the bit energy consumption of CSMA/CA was $4.9 \mu \mathrm{J}$ and the bit energy consumption of TDMA was $2.1 \mu \mathrm{J}-\mathrm{a}$ difference of $2.8 \mu \mathrm{J}$. Use of TDMA in IBC has the lowest energy consumption with the maximum value being nearly $2.3 \mu \mathrm{J} / \mathrm{bit}$ at 15 sub-nodes.

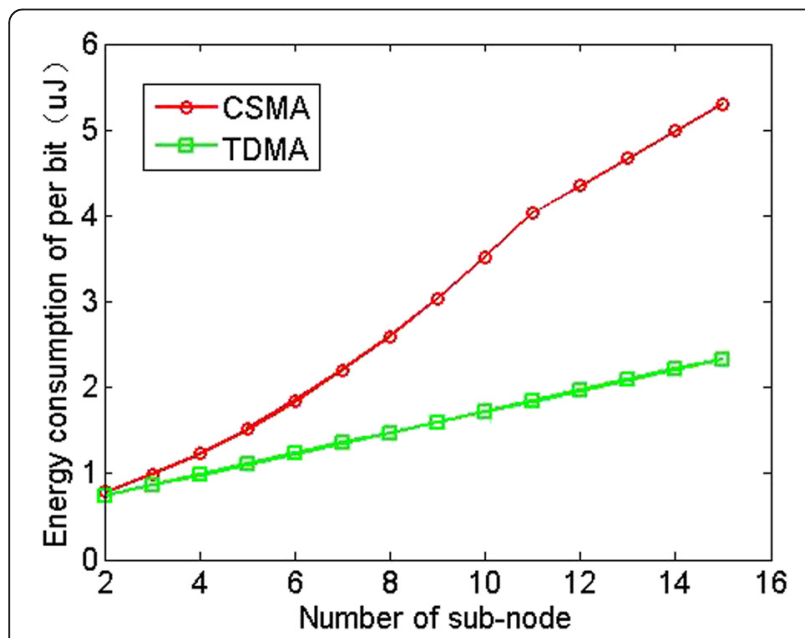

Fig. 10 Energy consumption comparisons of TDMA and CSMA/CA under the multi-node system
All in all, the bit energy consumption of TDMA is obviously lower than the bit energy consumption of CSMA/CA. The reason is that the CSMA/CA access mechanism with larger energy consumption competition, channel and node listens, and packet collisions consumes more network energy. CSMA/CA, however, has balanced each node in the channel, thus increasing the flexibility of the system. Nevertheless, considering its lower power consumption, TDMA may be more suitable for a BAN that is based on IBC.

\section{Conclusions}

In this research, to obtain the attenuation properties of IBC, in vivo experiments with 7 volunteers were carried out in a frequency band range from $10 \mathrm{kHz}$ to $500 \mathrm{kHz}$, and the results have indicated that human body channel attenuation can be equivalent to a band-pass filter characteristic. In addition, the equalizer which effectively reduced ISI was designed according to the frequency-selective fading characteristic of the human body channel. Then an IBC base band modulation simulation model was established based on human body channel characteristic, and the BER of different modulation methods were also evaluated and compared with each other. The results indicated that when higher band utilization was not required, FSK had lower BER and could better conform to the requirements of an IBC system. Moreover, FSK would not easily be affected by a change of channel parameters. To investigate the energy consumption of the MAC layer based on TDMA and CSMA/CA, a multi-node physiological signal monitor and transmission system was designed using the proposed band-pass filter characteristics of the human body to compare the power consumption of MAC layer based on TDMA and CSMA/CA. An FSK modulationdemodulation method was applied in the system. The outcome showed that TDMA had lower power consumption, but the number of nodes was easier to change under CSMA/CA because each node had the same priority. Considering the different priorities of human physiological signals and the lower power consumption, an FSK method with TDMA is more suitable for human communication in health care systems.

Competing interests

The authors declare that they have no competing interests.

\section{Acknowledgements}

This work was supported by the Project of Chinese Ministry of Science and Technology 2013DFG32530, the National Natural Science Foundation of China U1505251 and 61201397, the Funds of the Department of Education of Fujian Province, China, JA13027 and the Project of the Department of Education of Fujian Province, China,JK2014001.

\section{Author details}

${ }^{1}$ College of Physics and Information Engineering, Fuzhou University, Fuzhou, China. ${ }^{2}$ Key Lab of Medical Instrumentation \& Pharmaceutical Technology of 
Fujian Province, Fuzhou, China. ${ }^{3}$ State Key Laboratory of Analog and Mixed Signal VLSI, University of Macau, Macau, China. ${ }^{4}$ Department of Electrical and Computer Engineering, Faculty of Science and Technology, University of Macau, Macau, China.

Received: 28 January 2016 Accepted: 4 April 2016

Published online: 18 April 2016

\section{References}

1. IEEE 802.15 is the Working Group for WPAN \& Task Group 6 is the group responsible for BANs. http://www.ieee802.org/15/pub/TG6.html.

2. P Honeine, F Mourad, M Kallas et al., Wireless sensor networks in biomedical: Body area networks, Systems, Signal Processing and their Applications (WOSSPA), 2011 7th International Workshop on, 2011, pp. 388-391

3. IEEE Standards Association. 802.15. 6-2012 IEEE Standards for Local and Metropolitan Area Networks-Part 15.6: Wireless Body Area Networks[]].

4. TG Zimmerman, Personal area networks: near-field intrabody communication[J]. IBM Syst. J. 35(3.4), 609-617 (1996)

5. JE Ferguson, AD Redish, Wireless communication with implanted medical devices using the conductive properties of the body[J]. Expert Rev. Med. Devices 8(4), 427-433 (2011)

6. SH Pun, YM Gao, PU Mak et al., Quasi-static modeling of human limb for intra-body communications with experiments[J]. Inform. Technol Biomed, IEEE Transact 15(6), 870-876 (2011)

7. Y-m Gao, P Siohang, D Min et al., Quasi-static model and transceiver design for galvanic coupling intra-body communication. J Electron. Meas. Instrum. 26(8), 732-737 (2012)

8. Y-m Gao, P Siohang, D Min et al., Model and analyze for the intra-body communication of the implantable medical sensors. Chin. J. Sci. Instrum. 33(12), 21-26 (2012)

9. M Amparo Callejon, D Naranjo-Hernández, J Reina-Tosina et al., Distributed circuit modeling of galvanic and capacitive coupling for intrabody communication [J]. Biomed. Eng., IEEE Trans. 59(11), 3263-3269 (2012)

10. K Hachisuka, Y Terauchi, Y Kishi et al., Simplified circuit modeling and fabrication of intrabody communication devices[J]. Sensors Actuators A Phys. 130, 322-330 (2006)

11. MS Wegmueller, M Oberle, N Felber et al., Signal transmission by galvanic coupling through the human body [J]. Instrum. Meas., IEEE Trans. 59(4), 963-969 (2010)

12. Ž Lucev, I Kroisd, M Cifrek, A capacitive intrabody communication channel from $100 \mathrm{kHz}$ to $100 \mathrm{MHz}$ [J]. Instrum. Meas., IEEE Trans. 61(12), 3280-3289 (2012)

13. N Haga, K Saito, M Takahashi et al., Equivalent circuit of intrabody communication channels inducing conduction currents inside the human body [J]. Antennas Propag, IEEE Trans 61(5), 2807-2816 (2013)

14. R Xu, WC Ng, H Zhu et al., Equation environment coupling and interference on the electric-field intrabody communication channel [J]. Biomed. Eng., IEEE Trans. 59(7), 2051-2059 (2012)

15. W Hao, CS Choy, A $170 \mathrm{~cm}$ transmission distance, high speed IntraBody Communication receiver design and its application to FPGA audio player[C]//TENCON 2013-2013 IEEE Region 10 Conference (31194). IEEE, 2013: 1-4

16. Changjiang Dua, Zedong Nie, et al. Audio Communication System: China, 103888194 A[P].2014.06.25.

17. N Zedong, G Feng, $\mathrm{H}$ Jin et al., Low power single-chip RF transceiver for human body communication [J]. China Commun. 9(9), 1-10 (2012)

18. XM Chen, PU Mak, SH Pun et al., Study of channel characteristics for galvanic-type intra-body communication based on a transfer function from a quasi-static field model [J]. Sensors 12(12), 16433-16450 (2012)

19. O Omeni, A Wong, AJ Burdett et al., Energy efficient medium access protocol for wireless medical body area sensor networks [J]. Biomed. Circuits Syst., IEEE Trans. 2(4), 251-259 (2008)

20. KS Prabh, F Royo, S Tennina, et al, BANMAC: an opportunistic MAC protocol for reliable communications in body area networks[C]//Distributed Computing in Sensor Systems (DCOSS), 2012 IEEE 8th International Conference on. IEEE, 2012: 166-175

21. S Hayat, N Javaid, ZA Khan, et al, Energy efficient MAC protocols[C]//High Performance Computing and Communication \& 2012 IEEE 9th International Conference on Embedded Software and Systems (HPCC-ICESS), 2012 IEEE 14th International Conference on. IEEE, 2012: 1185-1192
22. R Rosini, F Martelli, M Maman, et al, On-body area networks: from channel measurements to mac layer performance evaluation[C]//European Wireless, 2012. EW. 18th European Wireless Conference. VDE, 2012: 1-7

23. XZ Zeng, YM Gao, SH Pun, Effects of muscle conductivity on signal transmission of intra-body communications[J]. J. Electron. Meas. Instrum. 27(1),21-25 (2013)

24. D Hua, MATLAB simulation and communication application example explanation[M], (Posts \& Telecom Press, 2003)

25. S Chapman, MATLAB programming for engineers[M], (Nelson Education, 2015.)

26. J Proakis, M Salehi, G Bauch, Contemporary communication systems using MATLAB[M], (Cengage Learning, 2012)

27. ER Hapeman, WR Zeuch, JA Crandall et al., Telecom Glossary 2000-American National Standard T1, 2001, pp. 523-2001. http[J]

28. Z TANG, L FENG, D XIE et al., Analysis and research of volume conduction channel [J]. Comput. Simul. 2, 035 (2010)

29. Y Li, X Qi, M Keally et al., Communication energy modeling and optimization through joint packet size analysis of bsn and wifi networks [J]. Parallel Distrib. Syst., IEEE Trans. 24(9), 1741-1751 (2013)

30. G Zhou, Q Li, J Li et al., Adaptive and radio-agnostic qos for body sensor networks[J]. ACM Trans. Embed. Comput. Syst(TECS). 10(4), 48 (2011)

31. J Liu, Engineer experience-said STM32, (Beijing University of aeronautics and astronautics press, 2011)

\section{Submit your manuscript to a SpringerOpen ${ }^{\mathcal{O}}$ journal and benefit from:}

- Convenient online submission

- Rigorous peer review

- Immediate publication on acceptance

- Open access: articles freely available online

- High visibility within the field

- Retaining the copyright to your article

Submit your next manuscript at springeropen.com 